DRAFT VERSION NOVEMBER 8, 2018

Preprint typeset using $\mathrm{LT}_{\mathrm{E}} \mathrm{X}$ style emulateapj v. 08/22/09

\title{
PROSPECTS OF HIGH-RESOLUTION X-RAY SPECTROSCOPY FOR AGN FEEDBACK IN GALAXY CLUSTERS
}

\author{
S. HEINZ ${ }^{1}$, M. BRÜGGEN ${ }^{2}$, B. MORSONY ${ }^{1}$ \\ ${ }^{1}$ Astronomy Department, University of Wisconsin-Madison, 475. N. Charter St., Madison, WI 53706 and \\ ${ }^{2}$ Jacobs University Bremen, PO Box 750561, 28725 Bremen, Germany \\ Draft version November 8, 2018
}

\begin{abstract}
One of the legacies of the Chandra era is the discovery of AGN-inflated X-ray cavities in virtually all coolcore clusters, with mechanical luminosities comparable to or larger than the cluster cooling rate, suggesting that AGN might be responsible for heating clusters. This discovery poses a new set of questions that cannot be addressed by X-ray imaging or modeling alone: Are AGN actually responsible for halting cooling flows? How is the AGN energy transferred to heat? How tight is the observed balance between heating and cooling? Using numerical simulations and a new virtual X-ray observatory tool, we demonstrate that high-resolution, high-throughput X-ray spectroscopy can address these questions and that the International X-ray Observatory $I X O$ will have the necessary capabilities to deliver these measurements.

Subject headings: techniques:spectroscopic, X-rays:galaxies:clusters, ISM:kinematics and dynamics, galaxies:jes
\end{abstract}

\section{INTRODUCTION}

One of the surprising legacies of the Chandra era is the discovery of X-ray cavities in virtually every cool core galaxy cluster (e.g. Bîrzan et al. 2004; Allen et al. 2006). This led to the suggestion that AGN heating is responsible for halting the cooling in clusters and keeping cooling flows from actually reaching star-forming temperatures.

The discovery of the cavities allowed estimating the kinetic power of a large sample of radio galaxies, to within about an order of magnitude. The remaining uncertainties result from the fact that the cavity age cannot be determined from imaging studies alone. A range of age estimates have been used in the literature, ranging from the sound crossing time to the buoyant rise time (e.g. McNamara et al. 2000; Fabian et al. 2000; Churazov et al. 2001). Additionally, the orientation of the cavities relative to the line-of-sight cannot be determined from imaging observations alone, leading to an additional uncertainty in cavity size and age (e.g. Enßlin \& Heinz 2002). Finally, given that we do not know the inflation history of cavities, it is not clear how much energy has to be expended by the black hole to inflate a cavity to a given size.

Thus, while it is now generally accepted that AGN can release enough energy in principle to heat clusters (at least to within a factor of order unity), it is not clear whether and how this energy is transferred to the thermal gas in order to actually counteract the cooling.

To make progress, a quantitative spectroscopic approach is necessary. Unfortunately, neither Chandra nor XMM-Newton have the high spectral resolution and throughput required to observe the physical processes that control feedback. As we will show below, the requirements to solve the outstanding problems in cluster feedback posed by Chandra will be met by the International X-ray Observatory, IXO. Before presenting our simulations, it is worth reviewing the relevant technical specifications currently planned for IXO:

- Spectral resolution: IXO will feature an X-ray Microcalorimeter instrument $(X M S)$ with uniform $2.5 \mathrm{eV}$ spectral resolution up to $7 \mathrm{keV}$. At the $\mathrm{Fe} \mathrm{K} \alpha$ line en-

Electronic address: heinzs@astro.wisc.edu ergy of $\sim 6.7 \mathrm{keV}$, this corresponds to a spectral resolving power of 2700 , an improvement of roughly two orders of magnitude compared to the Chandra resolution for extended sources. The core $X M S$ array will have 3 arc-second pixels, arranged in a $40 \times 40$ square for a $2 \times 2$ arc-minute field-of-view.

- Effective area: Relative to Chandra imaging with the back-illuminated S3 chip, the effective area will be roughly a factor 25 higher, corresponding to the efficiency difference between the Keck telescopes and the Hubble Space Telescope HST.

- Angular resolution: The angular resolution of the IXO telescope mirror assembly will be 5 arc-second on-axis at $7 \mathrm{keV}$ (5 arc-second half-power diameter), which is very well suited for cluster studies. Again this is comparable to the angular resolution achievable by groundbased large telescopes compared to $H S T^{1}$.

The organization of this letter is as follows: In $\$ 2$ we describe the methods employed in the simulations presented in \$3. while $\$ 4$ discussed the consequences and $\$ 5$ presents our conclusions. Throughout this letter, we use concordance cosmological parameters of $H_{0}=70 \mathrm{~km} \mathrm{~s}^{-1} \mathrm{Mpc}^{-3}, \Omega_{\mathrm{m}}=0.3$, and $\Omega_{\Lambda}=0.7$ and cluster metallicities of 0.5 relative to solar.

\section{SIMULATIONS}

A powerful way to make detailed observational predictions of feedback is by constructing dedicated numerical simulations of radio galaxies in clusters and then virtually observing them with an X-ray telescope. This is the avenue chosen in this letter. Before presenting our results, we will briefly describe the method used.

\subsection{The code}

\footnotetext{
${ }^{1}$ While the 0.5 abscond Chandra resolution is necessary for the study of very fine details like the sound waves in Perseus, a fair fraction of cluster science enabled by Chandra could be done at 5 arc-second resolution.
} 
We use the publicly available FLASH code (Fryxell et al. 2000) which is a modular block-structured adaptive mesh refinement code. It solves the Riemann problem on a Cartesian grid using the Piecewise-Parabolic Method. Our simulation includes $7 \times 10^{5}$ dark matter particles. The particles are advanced using a cosmological variable-timestep leapfrogmethod. Gravity is computed by solving Poisson's equation with a multigrid method using isolated boundary conditions. Our simulations have an effective dynamic range between 10000 and 20000.

In order to simulate radio galaxies in clusters, we inject back-to-back supersonic jets by placing arbitrary inflowboundary conditions into the grid. The location of the "jet nozzle" tracks the dynamical center of the cluster potential. The jets are injected with a velocity of $0.1 \mathrm{c}$ and an internal Mach number of 37.

In order to reproduce the observed morphologies we impose a sub-grid jitter on the jet, confined to a 20 degree opening angle, known as the dentist drill effect (Scheuer 1982). More information on the numerical details of the type of simulations used in this letter are presented in Heinz et al. (2006).

\subsection{Initial conditions}

We used the S2 galaxy cluster from Springel et al. (2001) as our initial conditions. This is a cosmologically evolved rich cluster with a mass of $M \sim 10^{15} M_{\odot}$. We imported the initial conditions into FLASH and evolved the cluster for several dynamical times to eliminate any transients before setting off the jet.

The density of the $\mathrm{S} 2$ cluster is appropriate for all the clusters presented in this paper. The temperature profile is appropriate for our simulations of Cygnus A and Hydra A, but we had to adjust the temperature normalization for our simulations of the Perseus cluster, which has a moderately lower temperature.

Because the cluster is fully cosmologically evolved, our simulations incorporate a realistic level of density and temperature sub-structure, as well as a realistic cluster velocity field, including turbulence. We did not, however, include a sub-grid turbulence model.

\subsection{XIM : a virtual observatory}

The simulation output was then virtually observed using the publicly available in-house tool XIM (see Heinz \& Brüggen 2009). Taking input grids from numerical hydrodynamic simulations, XIM performs spectral modeling of thermal emission, including Doppler shifts and ionization balance. It then performs spectral projection along an arbitrary line-of-sight, PSF convolution, telescope and detector efficiency, and spectral convolution with the detector response (using the proper response files for current and future telescopes). Finally, it adds sky- and instrument backgrounds and Poisson counting error.

\subsection{Fe XXV as a kinematic tracer}

In this paper we concentrate on the $\mathrm{K} \alpha$ line from Heliumlike iron Fe XXV. This line complex is very luminous in the intra-cluster gas and because of the large mass of Fe relative to other abundant species, it is an excellent kinematic tracer (Brüggen et al. 2005).

Many other lines are present, also showing the kinematic signatures discussed below, but for simplicity and clarity we limited the analysis to Fe XXV. Figure 1 shows the rest-frame

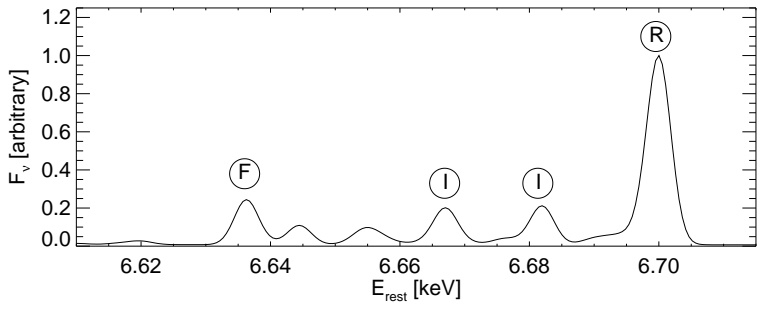

FIG. 1.- Reference spectrum of the Fe XXV K $\alpha$ line for a $4 \mathrm{keV}$ thermal plasma, with resonance, intercombination, and forbidden lines labeled. Dielectronic satellite lines are also included.

multiplet line structure at a plasma temperature of $4 \mathrm{keV}$ for reference. Given its strength, the resonance line at $6.7 \mathrm{keV}$ rest energy is the best suited for kinematic measurements.

\section{RESULTS}

To investigate the possibilities offered by high-throughput $\mathrm{X}$-ray spectrographs for the study of AGN feedback in clusters, we constructed virtual IXO observations of three representative (benchmark) galaxy clusters with detailed Chandra cavity studies: Perseus A, Cygnus A, and Hydra A. The simulations aim to reconstruct, as best as currently possible, the observational characteristic of these sources.

Before presenting the three cases in more detail, it is worth pointing out that $I X O$ data are difficult to visualize in 2D figures. The plots show different spectra from virtual spectral slits placed across that data cube, which clearly show the ability of $I X O$ to resolve the kinematic signatures of cluster feedback. However, a much better way to view the data is by animation. For this reason we have prepared movies of all three cases that can be viewed at http://www.astro.wisc.edu/ heinzs/feedbackmovies.html

\subsection{Perseus $A$}

The Perseus cluster was the first cluster known to have Xray cavities. With a mega-second of Chandra observing time, this is the best studied cluster to date, displaying rich morphological complexity across all wavebands (e.g. Graham et al. 2008; Fabian et al. 2008; Taylor et al. 2006). As such, it represents the benchmark for numerical models.

As a moderately powerful source, with a jet power somewhere between $10^{44}$ and $10^{45} \mathrm{ergs} \mathrm{s}^{-1}$, it also represents a good example of what has become known as "gentle" or "effervescent" heating (e.g. Ruszkowski et al. 2004).

The detection of a weak shock in the long Chandra image (Graham et al. 2008) has provided the most robust power estimate of $W_{\text {jet }} \sim 10^{45} \mathrm{ergs} \mathrm{s}^{-1}$, which is the number we chose to use. Given this power estimate, we ran the simulation for $10^{7}$ yrs until the size of the shock and cavities corresponded to the observed values.

The central cluster temperature is around 3-4 keV, somewhat colder than the S2 cluster we used as our initial condition. We thus adjusted the cluster temperature in postprocessing to ensure the correct line strengths and ratios, fixing the total X-ray power from the cluster to the observed Chandra flux.

The resulting simulated IXO image and Fe XXV K $\alpha$ spectrum are shown in Fig. 2 for an assumed exposure time of 250 $\mathrm{ksec}$, at a red-shift of $z=0.01756$. The cavities are clearly visible, as is the classical bright central core of the Perseus cluster.

Figure 2 also show the spectrum for a virtual spectral slit placed across both cavities (see image for slit placement). 

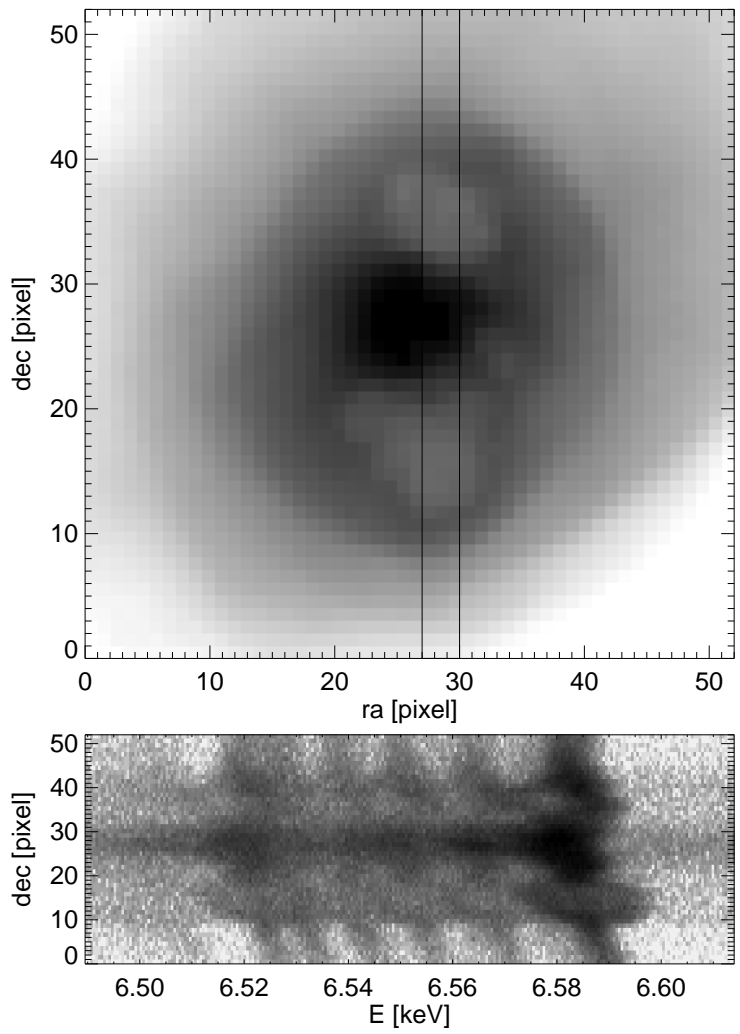

FIG. 2.- Virtual $250 \mathrm{ksec} I X O$ observation of Perseus cluster (redshift $z=0.01756$ ). Top: $0.5-10 \mathrm{keV}$ image. Bottom: Fe XXV K $\alpha$ spectrum of a 2-pixel vertical slit across the cavities, clearly showing the kinematic signature of the expanding bubble.

Apart from the complex sub-structure of the Fe XXV K $\alpha$ multiplet, the most striking feature immediately visible from the spectrum is how the lines splits at the locations of the cavities.

The two "bubbles" seen in the spectrum correspond to the front and back walls of the cavities, allowing full 3D reconstruction from the imaging spectrum like in the case of supernova remnants. Simply reading off the expansion velocity from the blue- and red-shifted line gives a line-of-sight velocity of $v_{\text {LOS }} \sim \pm 350 \mathrm{~km} \mathrm{~s}^{-1}$, compared to the actual velocity of $\sim 325 \mathrm{~km} \mathrm{~s}^{-1}$ in the simulation.

Thus, IXO will be able to clearly resolve the kinematic signature of expanding bubbles around cluster radio sources like Perseus A. It is also clear that angular resolution of a few arcseconds is vital to resolve the cavities both spectrally and spatially.

\subsection{Cygnus $A$}

As a powerful FR2 source, Cygnus A sits at the upper end of the expected range of cluster radio sources and shows clear cavities and possibly a shock in the Chandra image (Wilson et al. 2006). As with most cluster sources, the ambiguities in the interpretation of the imaging data cannot constrain the jet power to better than about an order of magnitude, roughly $10^{45}$ to $10^{46} \mathrm{ergs} \mathrm{s}^{-1}$.

The discovery of giant X-ray cavities in other clusters has shown that powerful outbursts like the one currently observed in Cygnus A might be more common than implied by models of "gentle" cluster heating, making this source an important benchmark.

Our initial numerical simulations of Cygnus A have been
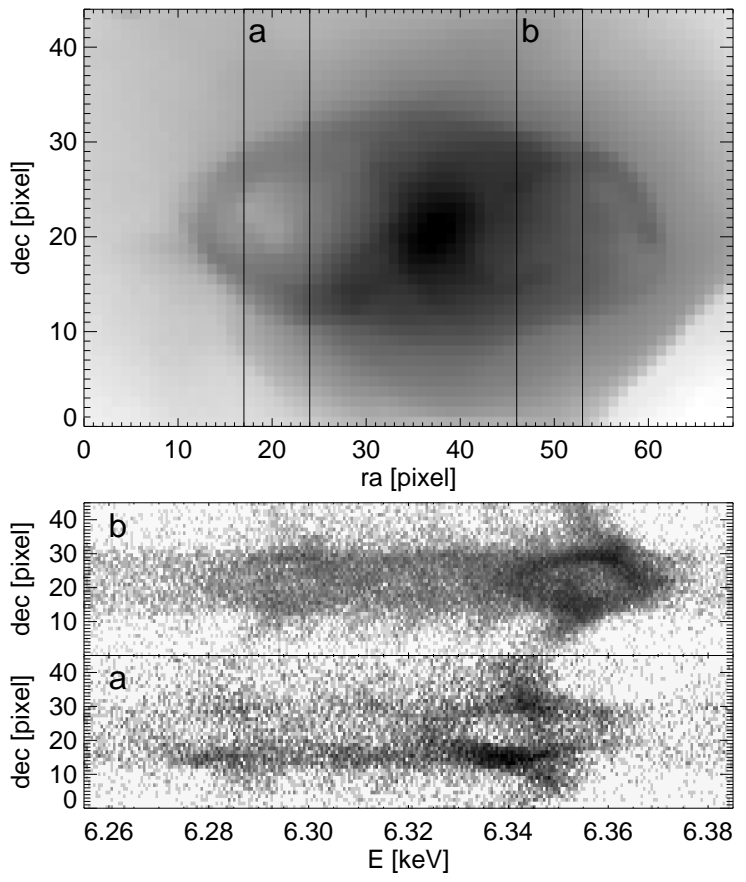

FIG. 3.- Virtual $250 \mathrm{ksec} I X O$ observation of Cygnus A (redshift $z=$ 0.056). Top: $0.5-10 \mathrm{keV}$ image. Middle: Fe XXV K $\alpha$ line of the eastern cavity. Bottom: same for the western cavity. Both cavities will be easily resolved by $I X O$.

presented in Heinz et al. (2006). Here we will present the virtual $I X O$ observation derived from that simulation. We ran the simulation with a jet power of $10^{46} \mathrm{ergs} \mathrm{s}^{-1}$ for $21 \mathrm{Myrs}$, at which point the simulation reached the observed cavity and radio lobe size and morphology. ${ }^{2}$

Our virtual $250 \mathrm{ksec} I X O$ observation is shown in Fig. 3, for a cluster redshift of $z=0.056$. The two bottom spectra show the FeXXV K-alpha line, clearly resolving the two cavities (the two spectra correspond to two virtual spectral slits, one for each cavity). As expected, for a source this powerful it will be easy for $I X O$ to resolve the kinematic structure of the line. Thus, IXO will be able to conclusively determine the jet power of Cygnus A.

\subsection{Hydra A}

Several clusters that currently contain relatively modest radio sources show fossil cavities that imply much more powerful past outbursts, most notably Hydra A (Wise et al. 2007), MS 0735 (Gitti et al. 2007), and Hercules A (Nulsen et al. 2005). Confirming this interpretation will be an important test of impulsive vs. gentle cluster heating models (note that Wise et al. (2007) identify multiple powerful outbursts in Hydra A).

We used a late timestep of our $10^{46} \mathrm{ergs} \mathrm{s}^{-1}$ simulation to represent this case, 150 Myrs after the outburst started, roughly corresponding to the case of Hydra A. The cavity sizes are of the same order as those observed in Hydra A, including the north-south asymmetry due to motion of the intracluster medium (ICM).

Given the low surface brightness this far out in the cluster

${ }^{2}$ We also tested the predicted virtual Chandra surface brightness of the simulation against the actual observation and found that they agree to within better than $10 \%$ in the region of the rim around the cavity, giving us confidence in the predicted line fluxes. 

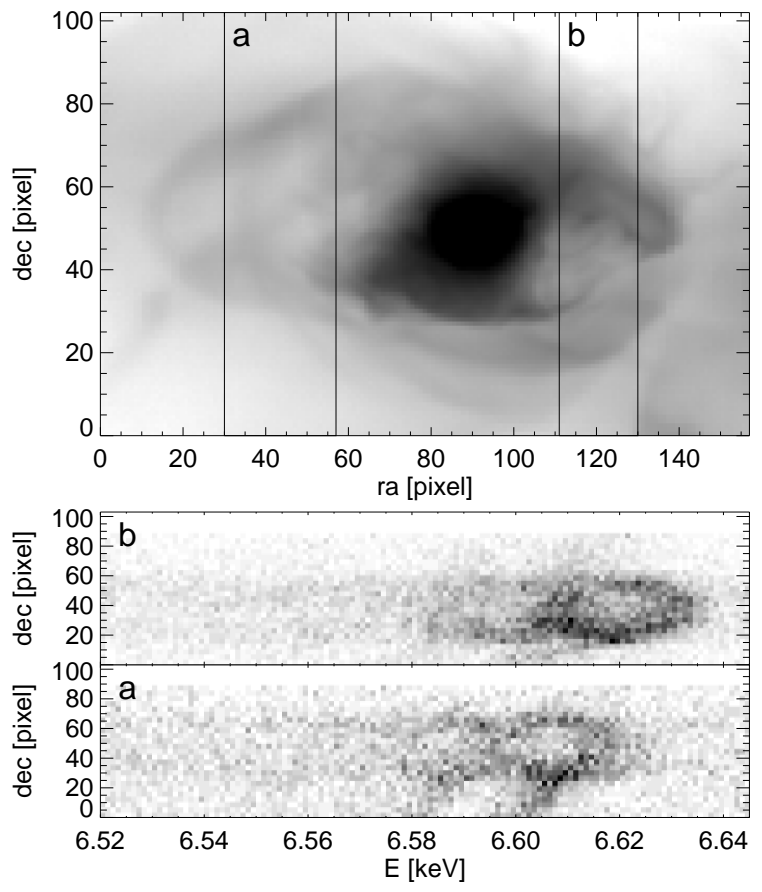

FIG. 4.- Virtual $250 \mathrm{ksec} I X O$ observation of a Hydra A-like radio source. Top: $0.5-10 \mathrm{keV}$ image. Middle and bottom: Fe XXV K $\alpha$ spectra of both cavities.

and the large angular size of these sources, such observations will be more challenging (requiring multiple long pointings). Still, the $250 \mathrm{ksec}$ virtual observation in Fig. 4) shows that IXO can resolve and detect the velocity structure of the cavities in these sources.

\section{DISCUSSION}

\subsection{Implications for other cluster radio sources}

The three detailed examples presented above show that a IXO will be able to resolve the kinematic structure of radio galaxy drive cavities for a significant fraction of the sources. In the case of Perseus $A$, the large count rate will allow a clear resolution of the iron line in observations as short as 20 ksec. This implies that even in clusters with lower surface brightness, IXO would be able to deliver cavity velocities in exposures of moderate length (100-200 ksec).

In principle, $I X O$ will be able to resolve cavities expanding with velocities as slow as $v_{\text {LOS }} \gtrsim 100 \mathrm{~km} \mathrm{~s}^{-1}$, though the presence of turbulent broadening in the ICM will likely mean a higher threshold (see 4.2 ). Given that the expected expansion velocity of actively jet-driven cavities for a fixed physical cavity radius $R$ scales like (Heinz et al. 1998)

$$
v_{\mathrm{LOS}} \propto\left(\frac{W_{\mathrm{jet}}}{\rho_{\mathrm{ICM}}}\right)^{1 / 3}
$$

low-power sources like M87 would be more difficult to observe. Detailed predictions of what $I X O$ would observe in the case of M87 will have to await more dedicated simulations.

\subsection{Caveats}

Given that the presented virtual observations are based on numerical simulations, there is are several physical effects that we did not include and that could in principal affect the predicted results. However, the point of this paper is not the precise prediction of velocities of individual sources but rather to demonstrate that $I X O$ will be able to measure the expansion rates for the expected range of velocities. We will therefore not go into any detail of the numerical shortcomings (like the absence of magnetic fields, the limitation to non-relativistic simulations, the restriction to purely thermal particle populations, and neglecting effects of radiative transfer like resonant scattering).

One of the critical elements that should be addressed is the level of turbulence present in the ICM significant turbulence will broaden all lines and make kinematic measurements of the sort discussed here more difficult (Inogamov \& Sunyaev 2003; Churazov et al. 2004). While our simulations do include turbulence on the scales resolved by our grid, we cannot make any statements regarding the level of sub-grid turbulence. Turbulent velocities in excess of $300 \mathrm{~km} \mathrm{~s}^{-1}$ will impede cavity measurements.

Finally, calibration uncertainties present in any X-ray instrument will in principle affect the spectroscopic accuracy. However, because we are only discussing kinematic effects, systematic errors due to calibration errors should not have any strong effect on our results.

\subsection{Other prospects for feedback studies}

The analysis presented above is only the most forward and simple application of high-resolution, high-throughput X-ray spectroscopy to the questions of cluster feedback. Given the spectral complexity of typical thermal spectra, many more applications will be possible that are well beyond the scope of this letter. We will suggest a few avenues that promise to be particularly fruitful:

- Line stacking: Given IXO 's high spectral resolution over a wide bandwidth, it will be easy to re-grid the spectral data to a logarithmic grid, upon which one can stack different emission lines on top of each other simply by shifting along the energy axis, greatly increasing signal-to-noise (thus reducing the overall exposure time requirements). More complex deconvolution algorithms could yield even more powerful diagnostics, including the temperature structure of the cavity shells from line-ratio variations.

- Line-of-sight angles: From the velocity centroid of the two cavities, relative to the cluster mean, it will be possible to determine the mean jet orientation relative to the line-of-sight as well as detecting large scale rotation in the cluster.

- Emission measure mapping: Beyond simple kinematics, spectral fitting will allow the extraction of the emission measure distribution in each individual X-ray pixel, allowing multi-phase metallicity, entropy, temperature, and pressure maps. The ultimate promise of this approach is the direct detection of the spectroscopic signature of AGN heating. The performance requirements on the $X M S$ calibration to allow such a measurement has yet to be formulated.

\section{CONCLUSIONS}

We showed that the quantitative kinematic measurements critical for establishing accurate cavity ages, jet powers, and black hole duty cycles are only achievable through highresolution X-ray spectroscopy. Our simulations demonstrate 
that the $2.5 \mathrm{eV}$ spectral resolution and 5 arc-second spatial resolution provided by the International X-ray Observatory $I X O$ will be sufficient to make the necessary measurements. Any significant compromise in angular or spectral resolu- tion would render these measurements impossible, implying that neither the current or any other planned X-ray telescopes (such as Astro- $H$ ) could deliver them instead of IXO .

\section{REFERENCES}

Allen, S. W., Dunn, R. J. H., Fabian, A. C., Taylor, G. B., \& Reynolds, C. S. 2006, MNRAS, 372, 21

Bîrzan, L., Rafferty, D. A., McNamara, B. R., Wise, M. W., \& Nulsen, P. E. J. 2004, ApJ, 607,800

Brüggen, M., Hoeft, M., \& Ruszkowski, M. 2005, ApJ, 628, 153

Churazov, E., Brüggen, M., Kaiser, C. R., Böhringer, H., \& Forman, W. 2001, ApJ, 554, 261

Churazov, E., Forman, W., Jones, C., Sunyaev, R., \& Böhringer, H. 2004, MNRAS, 347, 29

Enßlin, T. A. \& Heinz, S. 2002, A\&A, 384, L27

Fabian, A. C. et al. 2008, Nature, 454, 968

Fabian, A. C. et al. 2000, MNRAS, 318, L65

Fryxell, B. et al. 2000, ApJS, 131, 273

Gitti, M., McNamara, B. R., Nulsen, P. E. J., \& Wise, M. W. 2007, ApJ, 660, 1118

Graham, J., Fabian, A. C., \& Sanders, J. S. 2008, MNRAS, 386, 278

Heinz, S. \& Brüggen, M. 2009, submitted to ApJS, ArXiv e-prints
Heinz, S., Brüggen, M., Young, A., \& Levesque, E. 2006, MNRAS, in press Heinz, S., Reynolds, C. S., \& Begelman, M. C. 1998, ApJ, 501, 126 Inogamov, N. A. \& Sunyaev, R. A. 2003, Astronomy Letters, 29, 791

McNamara, B. R. et al. 2000, ApJ, 534, L135

Nulsen, P. E. J., Hambrick, D. C., McNamara, B. R., Rafferty, D., Birzan, L., Wise, M. W., \& David, L. P. 2005, ApJ, 625, L9

Ruszkowski, M., Brüggen, M., \& Begelman, M. C. 2004, ApJ, 611, 158 Scheuer, P. A. G. 1982, in IAU Symp. 97: Extragalactic Radio Sources, ed.

D. S. Heeschen \& C. M. Wade, 163-165

Springel, V., White, M., \& Hernquist, L. 2001, ApJ, 549, 681

Taylor, G. B., Gugliucci, N. E., Fabian, A. C., Sanders, J. S., Gentile, G., \& Allen, S. W. 2006, MNRAS, 368, 1500

Wilson, A. S., Smith, D. A., \& Young, A. J. 2006, ApJ, 644, L9

Wise, M. W., McNamara, B. R., Nulsen, P. E. J., Houck, J. C., \& David, L. P. 2007, ApJ, 659, 1153 